BULLETIN OF THE

AMERICAN MATHEMATICAL SOCIETY

Volume 81, Number 3, May 1975

\title{
AN ATTACK ON RIGIDITY. I, II
}

\author{
BY ROBERT CONNELLY 1 \\ Communicated by Harry Kesten, December 31, 1974
}

1. Introduction. We are primarily interested in the continuous rigidity (as opposed to the infinitesimal rigidity) of polyhedral surfaces in three-space. In 1813 Cauchy proved (with a few patchable mistakes) that any two convex polyhedral surfaces that are isometric are congruent, and even today his result has been improved only infinitesimally. If one looks at strictly convex embeddings this implies that they are rigid (see Gluck [5] for definitions). However, since Cauchy, most efforts have been devoted to either infinitesimal rigidity (see Dehn [4] for instance) or uniqueness of embeddings in a class closely resembling convex embeddings (cf. Stoker [9], Alexandrov [1], or Pogorelov [8]) even in the smooth category, where more "modern" methods have supplanted Cauchy's (see Nirenberg [7], Chern [3], or Herglotz [6] for instance). These techniques seem not too promising for the old conjecture that all embedded (or immersed) polyhedral (or smooth) surfaces are continuously rigid.

We present some ideas and techniques which we hope will be useful for the more general rigidity problem. Among other things, we show that any embedded suspension of a polygonal circle is rigid, as well as Theorems 1 and 2 below.

2. The structural equations. We regard a polyhedron $P$ as a finite collection of points $p_{1}, p_{2}, \cdots$ in $\mathbf{R}^{3}$, together with certain unordered pairs of the points, which we call edges. If $P$ corresponds to a triangulated 2 -dimensional surface, then the subpolyhedron of points adjacent to a point $p$ corresponds to a circle. Let $p_{1}, p_{2}, \cdots, p_{n}$ denote those points as one proceeds cyclicly around the "link" of $p$. Let $e_{j}=p_{j}-p, j=1, \cdots, n$, be thought of as a vector. Choose some convenient reference vector, $R$, and let $\pi: \mathbf{R}^{3} \rightarrow R^{\perp}$ denote orthogonal projection onto the plane perpendicular to $R$. Let $\theta_{j j+1}$ denote the angle from $\pi\left(e_{j}\right)$ to $\pi\left(e_{j+1}\right)$. The idea is to write $\theta_{j j+1}$, or more conveniently $e^{i \theta_{j j+1}}$, as some reasonable function of $z_{j}=R \cdot e_{j}, z_{j+1}=R \cdot e_{j+1}$,

AMS (MOS) subject classifications (1970). Primary 53C40, 53A05, 53C99.

Key words and phrases. Rigidity, flexing, isometry, bending, simplicial complex, polyhedron, combinatorial manifold, piecewise-linear manifold.

1 This research was supported by National Science Foundation Grant GP-33960X. 
and $x=R \cdot R$. We assume that no $\pi\left(e_{j}\right)=0$. Then it is not hard to show that

$$
e^{i \theta} j j+1=\left(Q_{j j+1}+y_{j j+1}\right) / H_{j} H_{j+1},
$$

where

$$
\begin{array}{r}
Q_{j j+1}=x\left(e_{j} \cdot e_{j+1}\right)-z_{j} z_{j+1}, y_{j j+1}=|R|\left[e_{j}, e_{j+1}, R\right] i, \\
H_{k}^{2}=x\left(e_{k} \cdot e_{k}\right)-z_{k}^{2}, \quad k=j, j+1, \\
y_{j j+1}^{2}=-x \operatorname{det}\left(\begin{array}{ccc}
e_{j} \cdot e_{j} & e_{j} \cdot e_{j+1} & z_{j} \\
e_{j+1} \cdot e_{j} & e_{j+1} \cdot e_{j+1} & z_{j+1} \\
z_{j} & z_{j+1} & x
\end{array}\right),
\end{array}
$$

$[,$,$] is the triple scalar product. Thus since \sum_{j=1}^{n} \theta_{j j+1}=2 \pi \omega$, where $\omega$ is the winding number of the link about the line through $R$, we have

$$
1=\prod_{j=1}^{n} e^{i \theta} j j+1=\prod_{j=1}^{n} \frac{Q_{j j+1}+y_{j j+1}}{H_{j} H_{j+1}}
$$

Cross multiplying we have the "structural" equations,

$$
\prod_{j=1}^{n} H_{j}^{2}=\prod_{j=1}^{n}\left(Q_{j j+1}+y_{j j+1}\right)=\prod_{j=1}^{n}\left(Q_{j j+1}-y_{j j+1}\right) \text {. }
$$

Note that $x, z_{1}, \cdots, z_{n}$ are the only extrinsic variables in this equation. $e_{j} \cdot e_{j}, e_{j} \cdot e_{j+1}$ depend only on the intrinsic nature of $P$.

3. Suspensions. We specialize to the following polyhedron, which we call a suspension, $\Sigma$. There are two points $N, S$, the north and south poles, with a common link $p_{1}, \cdots, p_{n}$. Call the edges $e_{j}=p_{j}-N, e_{j}^{\prime}=S-p_{j}$, $e_{j j+1}=p_{j}-p_{j+1}=e_{j}-e_{j+1}, j=1,2, \cdots, n$. The edges from $p_{j}$ to $p_{j+1}$ are called the equator.

Now we define $R=S-N$. It is easy to show

$$
R \cdot e_{j}=z_{j}=1 / 2\left(x+e_{j} \cdot e_{j}-e_{j}^{\prime} \cdot e_{j}^{\prime}\right)
$$

Thus the structural equation (*) becomes (up to the sign of $y_{j j+1}$ ) a function of $x=R \cdot R$.

If $\Sigma$ flexes (i.e. it is nonrigid) and $x$ is constant during the flex, it is easy to see that $\Sigma$ is not an immersion at either $N$ or $S$. So we assume that $x$ varies during a flex, so that all the quantities in $(*)$ become analytic functions of $x$ 
and are in fact defined for all complex $x$ over an appropriate Riemann surface say. Since all the quantities agree on the same small interval for real $x$, they agree everywhere. In particular they agree at $\infty$. We state one consequence of this technique.

THEOREM 1. Let $\Sigma$ flex with variable $x$. Then the winding number $\omega$ of the equator about the line through $N, S$ (which is defined for all $x$ in the interior of a flexing interval) is zero.

For any oriented polyhedral surface $P$, define a number

$$
V(P)=\sum\left[p_{i}, p_{k}, p_{l}\right] / 6,
$$

where $\left\langle p_{j}, p_{k}, p_{l}\right\rangle$ is an oriented triangle of $P$, and the summation is taken over all such triangles. If $P$ is an embedded surface, or is immersed bounding an immersed 3-manifold, then $V(P)$ is the volume of the bounded 3-manifold, if the proper orientation is chosen. Using techniques similar to those described above we show:

THEOREM 2. Let $\Sigma$, a suspension, flex with variable $x$. Then $V(\Sigma)=0$.

COROLlARY. If a suspension $\Sigma$ is embedded, or is immersed bounding an immersed 3-manifold, then $\Sigma$ is rigid.

4. Generalizations. It is possible to generalize Theorem 1 to the case when $\Sigma$ is a suspension of a piecewise $C^{1}$ curve (rather than piecewise-linear) by using somewhat different techniques. The structural equation used differs from (*) by a log, and an integral instead of a product is involved. However, the same principle of analytic continuation is still used.

It is also possible to use $(*)$ to give a fairly complete description of all flexible octahedra (and to some extent all suspensions as well). This involves the well-known group action on the nonsingular cubic $y^{2}=x\left(x-b^{\prime}\right)(x-b)$, $b, b$ real, and a flow graph used to describe $(*)$.

ADDED IN PROOF. We have recently found an example of an immersed polyhedral surface which flexes, contradicting one of the conjectures mentioned above. The conjecture, that all immersed polyhedral surfaces that bound an immersed 3-manifold are rigid, has not been contradicted and seems more appropriate.

We are also grateful to Branko Grünbaum for informing us of the work of Bricard [10], (and related references [11], [12], [13]), who gave a description of the flexible octahedra in 1897 ! 


\section{REFERENCES}

1. A. D. Alexandrov, Convex polyhedra, GITTL, Moscow, 1950; German transl., Math Lehrbücher und Monographien. Forschungsinstitut für Math. II. Abteilung: Math. Monographien, Band VIII, Akademie-Verlag, Berlin, 1958. MR 12, 732; 19, 1192.

2. A. Cauchy, Sur les polygones et polyedres, Second Mémoire, J. École Polytechnique 9 (1813).

3. S. S. Chern, Topics in differential geometry, Inst. for Advanced Study, Princeton, N. J., 1951. MR 19, 764.

4. M. Dehn, ̈̈ber die Starrheit konvexe Polyeder, Math. Ann. 77 (1916), 466-473.

5. H. Gluck, Almost all simply connected closed surfaces are rigid (preprint).

6. G. Herglotz, Über die Starrheit der Eiflächen, Abh. Math. Sem. Univ. Hamburg 15, (1943), 127-129. MR 7, 322.

7. L. Nirenberg, Rigidity of a class of closed surfaces, Nonlinear Problems (Proc. Sympos. Madison, Wis., 1962), Univ. of Wisconsin Press, Madison, Wis., 1963, pp. 177193. MR 27 \#697.

8. A. V. Pogorelov, Extrinsic geometry of convex surfaces, "Nauka", Moscow, 1969; English transl., Transl. Math. Monographs, vol. 35, Amer. Math. Soc., Providence, R. I., 1973. MR 39 \#6222.

9. J. J. Stoker, Jr., Geometrical problems concerning polyhedra in the large, Comm. Pure Appl. Math. 21 (1968), 119-168. MR 36 \#5815.

10. R. Bricard, Mémoire sur la theórie de l'octaèdre articulé, J. Math. Pures Appl. (5) (1897), $113-148$.

11. G. T. Bennett, Deformable octahedra, Proc. London Math. Soc. (2) 10 (1912), $309-343$.

12. A. Kokotsakis, ̈̈ber bewegliche Polyeder, Math. Ann. 107 (1933), 627-647.

13. H. Lebesgue, Octaèdres articulés de Bricard, Enseignement Math. (2) 13 (1967), 175-185 (1968). MR 38 \#2664.

DEPARTMENT OF MATHEMATICS, CORNELL UNIVERSITY, ITHACA, NEW YORK, 14850 